\title{
Pre-Treatment Dose Verification of Imrt Using Gafchromic Ebt3 Film and 2D- Array
}

\author{
Nalbant Nalbant, Donmez Kesen $\mathbf{N}$ and Bilge Hatice*
}

Radiation Oncology Department, Istanbul University Oncology Institute, Turgut Ozal Street Capa, Istanbul, Turkey

*Corresponding author: Bilge Hatice, Radiation Oncology Department, Istanbul University Oncology Institute, Turgut Ozal Street Capa, Istanbul, Turkey, Tel: + 902124142434, +905369167516; E-mail: nazo94@gmail.com

Received date: Jun 15, 2014, Accepted date: Jul 02, 2014, Publication date: Jul 25, 2014

Copyright: (c) 2014 Nalbant N, et al. This is an open-access article distributed under the terms of the Creative Commons Attribution License, which permits unrestricted use, distribution, and reproduction in any medium, provided the original author and source are credited.

\begin{abstract}
Purpose: The present work deals with pre-treatment dosimetric verification of 10 prostate intensity-modulated radiotherapy (IMRT) plans obtained by Gafchromic EBT3 film and 2D-Array seven29 to establish quality control protocol for the delivery of prostate IMRT in our clinic.

Methods: 10 prostate patients were irradiated according to IMRT techniques with 6 MV photon beams produced by a Varian DHX linear accelerator. Dose plans were computed using Eclipse 8.9 (version8.9, Varian, Palo Alto, CA, United States) treatment planning system (TPS). Pre-treatment dosimetric verification was carried out on field-perfield and total IMRT plan basis measuring 2D dose distributions in RW3 solid water phantom (PTW-Freiburg, Germany). For 10 patient plans, the dose distribution was re-calculated with the phantom CT scan and delivered to the phantom with the original and 0 degrees gantry, collimator and table angles. Thus IMRT quality control (QC) plans were generated. Dose distribution measurements were measured with Gafchromic EBT3 film and 2D-Array seven29 (PTW-Freiburg, Germany) two dimensional ionization chamber system. Verification measurements of total IMRT plans and each individual beams were compared with expected dose maps, and differences were evaluated by Verisoft program (PTW-Freiburg, Germany). To provide comparisons of multidimensional dose distributions, dose comparison tools such as gamma dose distribution, distance- to- agreement (DTA) and dose difference (DD) have been developed. The gamma dose distribution tool was used in our study. Three different gamma criteria of dose difference (DD) and distance to agreement (DTA) $(3 \% / 3 \mathrm{~mm}, 4 \% / 4 \mathrm{~mm}$ and $5 \% / 5 \mathrm{~mm}$ DD / DTA) are selected. These criteria are evaluated while suppressing the dose of $5 \%$ from dose distribution. Criterion validity accepted as section with gamma value less than or equal to $1(\gamma \leq 1)$ to be $90 \%$.
\end{abstract}

Results: In the comparison of dose distributions obtained from TPS and the results of PTW 2D-Array seven29 and Gafchromic EBT3 film dosimetry systems, it was showed that the compatibility of both methods were above $90 \%$ with respect to $3 \%$ DD and 3mm DTA criteria. PTW 2D-Array seven29 results compared to the results of the film was closer to the TPS data and this difference was statistically meaningful $(p<0.05)$. If the range of criteria is increased, the compatibility increases and it reaches to $100 \%$. It was observed that analyzing by measuring all fields which are composing IMRT plans and analyzing by measuring each individual fields at the same time, made no statistically meaningful difference $(p>0.05)$.

Conclusions: The measurements performed with PTW 2D-Array seven29 were closer to the TPS data than film measurements and it takes less time during clinical use, so it could be preferred for routine use. The present results suggest the gamma criteria of $3 \% / 3 \mathrm{~mm}$ as the most suitable criteria for prostate IMRT quality assurance.

Keywords: IMRT; Gafchromic EBT3 film; 2D-Array seven29; RW3 solid water phantom; Gamma analysis

\section{Introduction}

Intensity modulated fields have the potential to deliver optimum dose distributions which results in greater dose uniformity in the target and lower doses to the critical organs and normal tissue as compared to conventional radiotherapy. When is used Intensity modulated radiation therapy (IMRT), the dose distribution calculated by the treatment planning system (TPS) has to be verified before the treatment. The option of the TPS that permits exporting the true radiation fields to a quality control (QC) phantom [1,2]. There are several methods to verify the dose distribution calculated using two dimensional ionization chamber systems, electronic portal dosimetry
(EPID) and film dosimetry [3-7]. Film dosimetry is a well-established method to verify dose distribution in phantoms. It has high spatial resolution but needs long calibration procedures. For this reason, it is used for relative dose distribution. Recently, radiochromic films are used to provide treatment dose verification because of their low energy dependence. EBT3 films are used since 2011. It has same chemical composition of the active layer as EBT2 but featuring symmetrical construction for enhanced ease of use and accuracy [8,9]. Also, two dimensional (2D) detector arrays have become increasingly popular due to their ease of use and quick read of the results. QC phantom plan is generated where each beam is delivered perpendicularly to a flat surface solid phantom [10,11]. Since IMRT is more complex treatment. Dose fluencies obtained from TPS can be measured with these measuring systems and evaluated in analysis programs due to \% Dose Difference (DD) and Distance to Agreement (DTA) criteria or 
Gamma Index. These methods have been developed and implemented commercially [12-14].

In this work, EBT3 film and the seven29 2D array detector were used for IMRT pre-treatment verification. Furthermore, verification measurements of dose distribution were made for total IMRT plans and for each individual beam and compared with dose distribution from TPS.

It was aimed to suggest the faster and more useful dosimetric method for IMRT QA process and which gamma criteria is convenient to evaluate the treatment plans so establish a protocol for prostate IMRT technique in our institute.

\section{Method and Materials}

\section{QA tools}

The dose measurements were done in RW3 (PTW-Freiburg, Germany) parallel slabs of water equivalent phantom, PTW 2D-array seven29 dosimetry systems and Gafchromic EBT3 Film. The PTW RW3 water equivalent phantom dimensions are $30 \times 30 \times 30 \mathrm{~cm}^{3}$.

PTW 2D-Array seven29 is a system which is developed for dosimetric control of IMRT plans. Maximum measuring size is $27 \times 27 \mathrm{~cm}^{2}$. It is composed of 729 vented cubic ionization chamber with size of $5 \times 5 \times 5 \mathrm{~mm}$ and the volume of ionization chamber is $0.125 \mathrm{cc}$. As the distance between ion chambers is $5 \mathrm{~mm}$, the distance between the centres of two ionization chambers is $10 \mathrm{~mm}$. Surface of the system is covered with PMMA. It has $3.2 \mathrm{~kg}$ weight and $22 \mathrm{~mm}$ height. Active measuring point of ionization is $7.5 \mathrm{~mm}$ below the surface and its resolution is $0.1 \mathrm{mGy}$. PTW 2D-Array seven 29 quality control device is shown in Figure 1 [15].

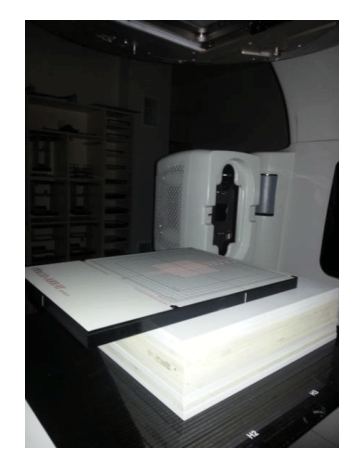

Figure 1: PTW 2D-Array seven29 quality control device.

Device is the one of the IMRT dosimetric quality control systems. Dose mapping measured with this system is compared with data obtained from TPS by using Verisoft program which performs gamma analysis by using dose difference (DTA) and percentage dose difference (DD\%) criteria. Regions which have rapid dose decrease can be measured with Verisoft program [16,17].

The Verisoft (PTW-Freiburg, Germany) software enables comparing dose distributions measured by some dosimetric systems and computed by radiotherapy treatment planning systems. The software uses the gamma evaluation method, it helps in locating hot and cold spots and determines maximum and average deviation between a calculated and measured plan.
Gafchromic $^{\bullet}$ EBT3 Film, (ISP-Asland, International Specialty Products, USA), is one of the dosimetry device used for quality control of patient plans. The most important characteristics of the film is to be positioned between 125 micron polyester layers, one on the top and one on the bottom, and this provides protection to 30 micron active layer against external factor. EBT3 film structure is shown in Figure 2.

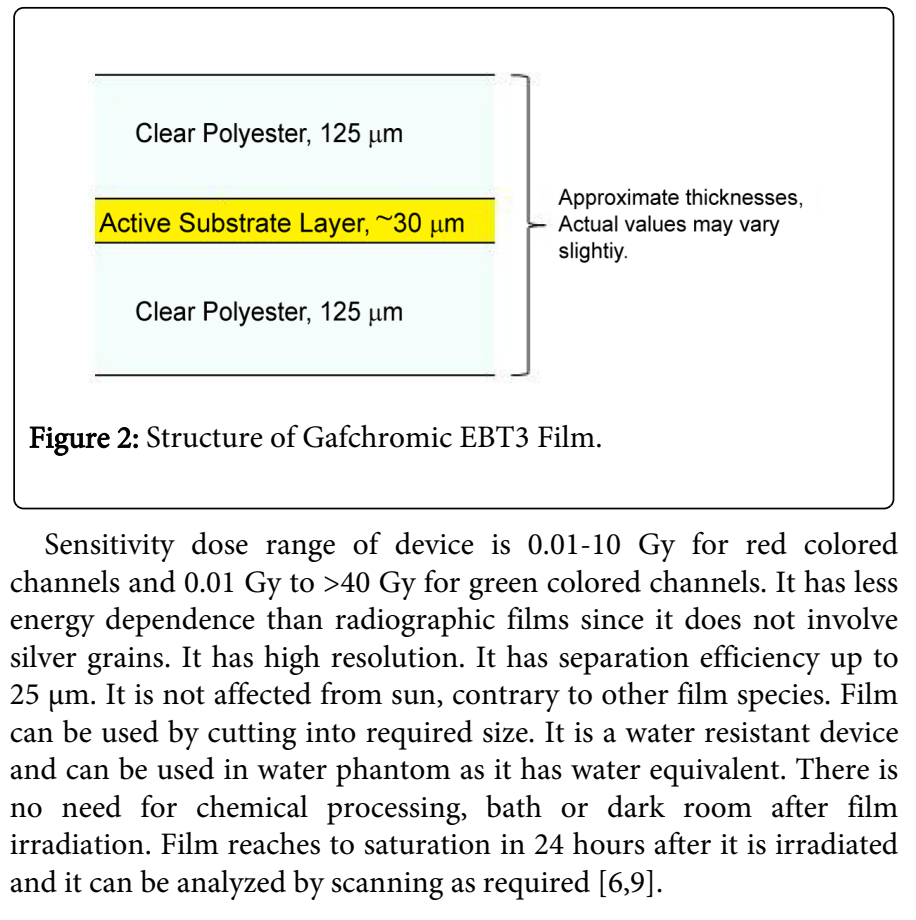

\section{Generation of IMRT Treatment Plans and Quality Control (QC) Plans}

This study included 10 patients with prostatic disease. The primary planning target volume (PTV56) contained only prostate and seminal vesicles -without lymph nodes- with a margin. The boost PTV (PTV75.6) contained only prostate volume with a margin. The prescription dose was 75.6 Gy (56 Gy to PTV and an additional 19.6 Gy to boost PTV; 2.1Gy/fraction) Treatment plans were generated in Varian Eclipse 8.9 (Varian Medical Systems, USA) TPS, by using AAA algorithm. Treatments were delivered with $6 \mathrm{MV}$ photon beams with a Varian DHX (Varian Medical Systems, USA) accelerator with 120-leaf millennium MLC. MLC leaf width is $5 \mathrm{~mm}$ at the central $20 \mathrm{~cm} ; 10$ $\mathrm{mm}$ at the outer $20 \mathrm{~cm}$ of the field. Varian specifies the leaf position end accuracy to be $1.0 \mathrm{~mm}$ and the end repeatability in millimeters at isocenter to be $0.6 \mathrm{~mm}$. The dose calculation grid was set to $2.5 \mathrm{~mm}$.

All patients were treated for prostate cancer, planned with a seven field dynamic IMRT technique. Plans with non-splitting fields in it were preferred as treatment plan option. Treatment plans of prostate patients who do not have wide irritated fields, were used. Only the prostate and seminal vesicle volumes are in the target, not the other lymph nodes. The treatment plan gantry angles were $0^{\circ}, 40^{\circ}, 80^{\circ}, 120^{\circ}$, $240^{\circ}, 280^{\circ}$, and $320^{\circ}$. Different PTV (planning target volume) volumes were irradiated with different doses (56 Gy, 75.6 Gy) simultaneously. In patients with appointed PTV56 (volume dose of $56 \mathrm{~Gy}$ ) and PTV75.6 (volume dose of $75.6 \mathrm{~Gy}$ ) volumes, centre of PTV75.6 volume was specified as isocentre. Required dose value of PTV volumes and tolerance value of critical organs were defined for optimization and IMRT plans were formed in the system. The 
planning goals were to cover $95 \%$ of the target volumes with $100 \%$ of the prescription dose and keep the critical structure doses at or below known tolerance limits. The goals for the rectum and bladder were to limit the volumes receiving more than $65 \mathrm{~Gy}$ and $40 \mathrm{~Gy}$ (V>65 Gy, $\mathrm{V}>40 \mathrm{~Gy}$ ) to $17 \%$ and $35 \% ; 25 \%$ and $50 \%$, respectively. The volume of bilateral femoral heads receiving $50 \mathrm{~Gy}$ was limited to less than $10 \%$ i.e. V50<10\% and $90 \%$ of penile bulb receiving dose $<15$ Gy was considered acceptable. Figure 3 shows IMRT plans generated in TPS.

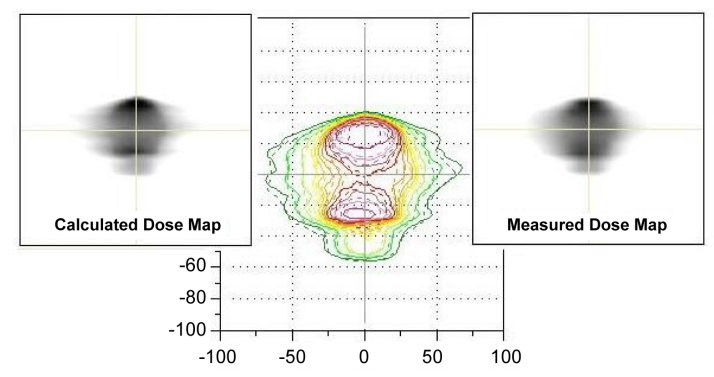

Figure 3: Calculated and measured dose fluence maps and their isodose comparison.

To arrange the quality control (QC) plans in TPS, a sandwich setup of water equivalent PTW RW3 plates with a stack of $10 \mathrm{~cm}$ below and $5 \mathrm{~cm}$ above the PTW 2D-array seven 29 were used.

The phantom arrangement was scanned in CT with slice thickness of $0.5 \mathrm{~cm}$. The scanned phantom is imported via DICOM to Eclipse treatment planning system (TPS). IMRT QC plans were generated by transferring each IMRT treatment plans to this setup assembly. The detectors have been employed for the evaluation of 70 intensity modulated radiation fields, were delivered with $6 \mathrm{MV}$ relative to ten patients.

All gantry, collimator and table angles were set to $0^{\circ}$. Thus planar dose distributions of total IMRT plans and each individual beams at 5 $\mathrm{cm}$ depth and with $95 \mathrm{~cm}$ SSD in a solid water phantom were obtained and saved for analyze.

\section{Measurement of IMRT Quality Control Plans with PTW 2D- Array seven29}

IMRT quality control plans of treatment plans which were prepared with Eclipse 8.9 software, were measured with Varian DHX linear accelerator at the same conditions with CT image, by using PTW 2DArray seven29. Isocentre was located on the top of ionization chambers in array. The measurements were performed in relative mode. Each field has been verified in solid water phantoms, with beam incidence perpendicular to the phantom surface. Planar dose distributions of total IMRT plans and each treatment fields, at $5 \mathrm{~cm}$ depth and with $95 \mathrm{~cm} \mathrm{SSD}$ in a solid water phantom with gantry, collimator and table angles set to $0^{\circ}$ were obtained with PTW 2DArray and compared with TPS calculated dose distributions under identical geometric setup. Measurement assembly consisting of PTW 2D-Array seven29 and PTW RW3 solid water phantom is shown in Figure 4 .

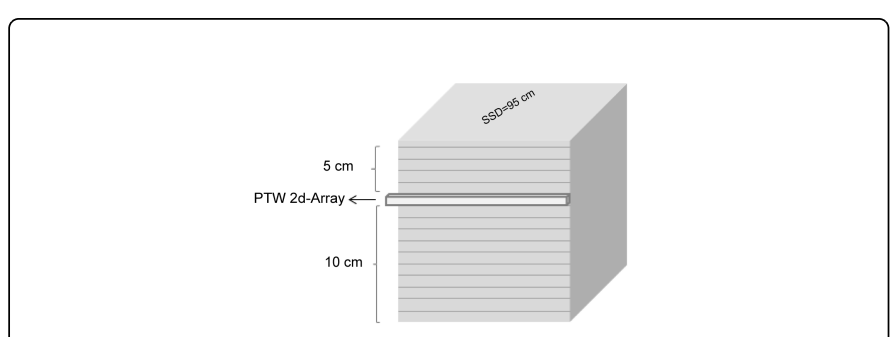

Figure 4: Solid water phantom and 2D-Array setup used for isodose distribution measurements.

\section{Measurement of IMRT Quality Control Plans with Gafchromic EBT3 Film}

In film dosimetry using radiochromic film and a flatbed scanner, the calibration curve is used to convert pixel values read out from the film to the dose. Before measuring, Gafchromic EBT3 films were prepared by cutting in size of $3 \times 3 \mathrm{~cm}^{2}$ for calibration curve used. Films were placed in $100 \mathrm{~cm} \mathrm{SAD}$ distance and at $5 \mathrm{~cm}$ depth and field size was set as $10 \times 10 \mathrm{~cm}^{2}$. For generation of calibration curve, irradiation was performed with dose values of $25 \mathrm{cGy}, 50 \mathrm{cGy}, 100 \mathrm{cGy}, 200 \mathrm{cGy}$, 300 cGy, 400 cGy, 500 cGy, 600 cGy, 700 cGy, 800 cGy, 1000 cGy and 1500 cGy. Exposed and an unexposed (background) films were scanned using a flatbed colour scanner (EPSON America Inc., Long Beach, CA) 24 hours later and analyzed using the ImageJ program; films were separated into three colors (red, blue and green). For optimum contrast, the red image was selected for film dosimetry, because the peak absorption of the EBT film is in the red region of the visible spectrum. The pixel values for unexposed (background) and exposed films were analyzed; analysis was performed over at the field centers using a region of interest (ROI) of $12.3 \times 12.3 \mathrm{~mm}^{2}$ and $1.23 \times 1.23 \mathrm{~mm}^{2}$, respectively. The calibration curve was formed with PTW FilmCal program (PTW-Freiburg, Germany) to calibrate the films with the curve and read the actual dose distributions by the film dosimetry. Film calibration curve is shown in Figure 5.

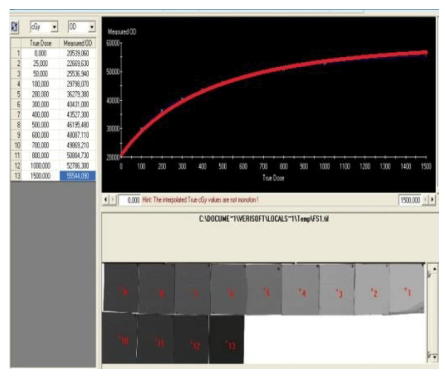

Figure 5: Film calibration curve was generated by films irradiated with different doses.

The measurements of 10 IMRT quality control plans were repeated by Gafchromic EBT3 Film at the same conditions with 2D-Array measurements.

For film dosimetry, measurement assembly consists of Gafchromic EBT3 Film and PTW RW3 solid water phantom. $5 \mathrm{~cm}$ RW3 solid water phantom was placed above, $10 \mathrm{~cm}$ RW3 solid water phantom 
below the film. Measurement was performed by coincide centre of film and RW3 solid water phantom, at $100 \mathrm{~cm}$ SAD distance.

Dose fluence maps of total IMRT plans and each treatment fields were saved individually for comparing with calculated dose distributions in TPS.

Comparisons between the PTW 2D-Array and Gafchromic EBT3 Film measured dose distributions and TPS calculated planar dose distributions were performed using Verisoft program with Gamma analyze method. Data from both detector array verification measurements were evaluated using gamma-index criteria of $3 \% / 3$ $\mathrm{mm}, 4 \% / 4 \mathrm{~mm}$ and $5 \% / 5 \mathrm{~mm}$ for local dose with a $>90 \%$ passing rate. It was performed by the suppression of the dose below of $5 \%$ from the maximum of the reference matrix. Wilcoxon Signed Ranks Test statistical method was used for result verification. The result was significant level for $\mathrm{p}<0.05$ value $[9,12]$.

\section{Results and Discussion}

Gamma analysis were carried out for total IMRT plans and each field 3\% DD and $3 \mathrm{~mm}$ DTA, 4\% DD and $4 \mathrm{~mm} \mathrm{DTA}$, and 5\% DD and $5 \mathrm{~mm}$ DTA criteria. Results were classified as TPS-2D Array and TPSEBT3 Film.

The gamma passed rates which obtained by comparisons of results calculated in TPS and total IMRT plans measured by PTW 2D Array seven29 ionization chamber system and Gafchromic EBT3 film in PTW RW3 solid water phantom are shown in Figure 6.

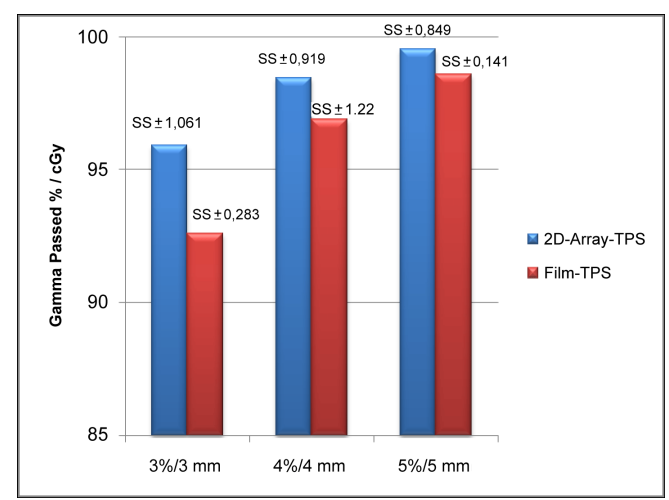

Figure 6: The gamma passed rates obtained by comparison of 2Darray and Gafchromic EBT3 Film results by TPS with different gamma criteria ( $\mathrm{SS}=$ standard deviation).

Fluence maps of total IMRT plan and each individual beams measured with 2D Array seven29 and Gafchromic EBT3 film were compared by calculated data in TPS. The gamma passed rates are shown in Figures 7 and 8.

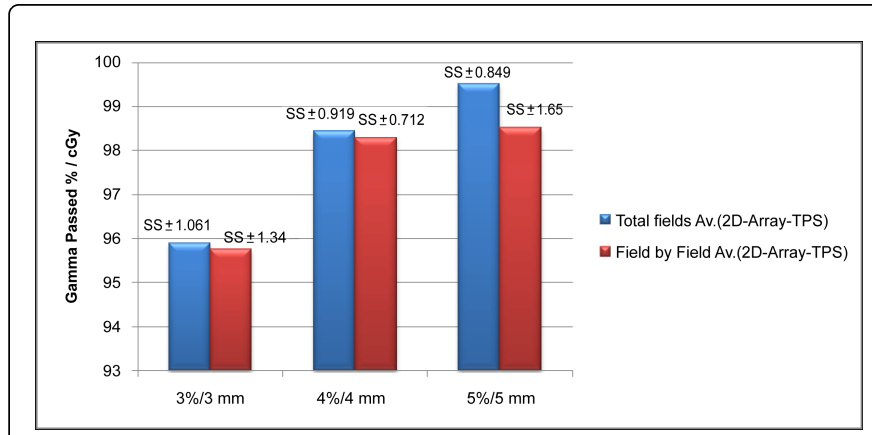

Figure 7: The gamma passed rates of total IMRT plan and each individual beams analyze methods obtained by comparison of 2Darray results by TPS with different gamma criteria ( $\mathrm{SS}=$ standard deviation).

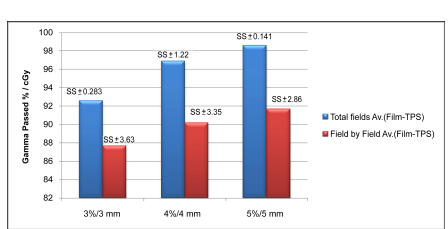

Figure 8: The gamma passed rates of total IMRT plan and each individual beams analyze methods obtained by comparison of Gafchromic EBT3 Film results by TPS with different gamma criteria (SS=standard deviation).

Due to complex nature of IMRT treatments, whether the treatment is delivered to linear accelerator as planned should be investigated before patients are treated. To serve this purpose, QC plans of prostate IMRT treatment plans were generated with two different dosimetric methods, -PTW 2D-Array seven29 two dimensional ionization chamber systems and Gafchromic EBT3 film dosimetry; both accuracy and applicability of used dosimetric control methods were researched.

Gamma analyze comparison values of total IMRT plans dose distributions obtained by calculating in TPS and measuring in PTW 2D-Array seven 29 were between $93 \%$ and $97.9 \%$, under $3 \%$ DD and 3 mm DTA criteria. As a result of comparisons of dose distributions obtained by TPS and Gafchromic EBT3 film, gamma values were found between $87.2 \%$ and $95.4 \%$ in the same gamma analyzes tolerance level. Differences between two methods was statistically significant $(\mathrm{p}<0.05)$.

For total IMRT plans, comparison results of dose distributions obtained by $2 \mathrm{D}$-Array seven 29 measurements and TPS calculations were between $97.1 \%$ and $100 \%$ under $4 \%$ and $4 \mathrm{~mm}$ gamma analyze tolerance level. As for the comparison of Gafchromic EBT3 film measurements and TPS calculations, gamma values were found between $94.2 \%$ and $96.88 \%$. Difference between two methods was statistically significant $(\mathrm{p}<0.05)$.

Gamma values obtained from the comparison of 2D-Array seven 29 and TPS were between $98.6 \%$ and $100 \%$ for $5 \%$ DD and $5 \mathrm{~mm}$ DTA tolerance limit in total IMRT plan irradiations. Gamma values obtained from the comparison of Gafchromic EBT3 film 
measurements and TPS calculations were between $97.4 \%$ and $99.5 \%$. Differences between two methods was statistically significant $(\mathrm{p}<0.05)$.

For total IMRT plans and each individual fields irradiations, under $3 \%$ DD and $3 \mathrm{~mm}$ DTA dose criteria, the averages of gamma analysis results obtained from comparison of TPS calculations and PTW 2DArray seven 29 measurements, were $95.9 \% 1.060$ and $95.76 \% \pm 1.34$, respectively. For $4 \% \mathrm{DD}$ and $4 \mathrm{~mm}$ DTA criteria, the averages of gamma values belong to the comparisons of TPS and PTW 2D-Array seven 29 , were $98.44 \% \pm 0.919$ and $98.29 \% \pm 0.712$; as for $5 \%$ DD and 5 mm DTA criteria, averages were $99.51 \% \pm 0.849$ and $98.52 \% \pm 1.65$ for two different quality control process. Differences between two methods was not significant $(\mathrm{p}<0.05)$.

When analyzing the comparisons of gamma values belong to the total IMRT plans and each individual fields calculated in TPS and measured by Gafchromic EBT3 film, under the gamma analyze criteria of $3 \% \mathrm{DD}$ and $3 \mathrm{~mm}$ DTA, the averages were found $92.60 \% \pm 0.283$ and $87.69 \% \pm 3.63$, respectively. Besides, under the $4 \%$ DD and $4 \mathrm{~mm}$ DTA gamma analyze criteria, averages were $96.88 \% \pm 1.22$ and $90.22 \%$ \pm 3.35 ; and for $5 \% \mathrm{DD}$ and $5 \mathrm{~mm}$ DTA gamma analyze criteria averages were $98.59 \% \pm 0.141$ and $91.69 \% \pm 2.86$, in the comparison of the gamma values belong to the total IMRT plan and each individual fields irradiations, respectively. Differences between two methods was not significant $(\mathrm{p}<0.05)$.

Llinares et al. [10] used PTW 2D-Array two dimensional ionization chamber system in PTW Octavius phantom and film for create IMRT plans in the gantry angles of 00, 500, 1000, 1500, 2100, 2600 and 3100. Gamma analyses were between $91.4 \%$ and $100 \%$ in the comparison of PTW 2D-Array seven 29 with TPS, for 3\% DD and $3 \mathrm{~mm}$ DTA criteria. In the dose fluence map comparison of TPS and film, results were found between $87.7 \%$ and $95.5 \%$.

Elawady et al. [18] reported, the agreement between measured with 2D-array and calculated doses for head and neck tumors using gamma criteria of $3 \%$ DD, $3 \mathrm{~mm}$ DTA and suppressing dose below $10 \%$, the average and standard deviation for local dose of gamma index are $81.70 \pm 6.28$, while those for maximum dose of gamma index are 92.48 $\pm 4.60$

When $4 \%$ DD, $4 \mathrm{~mm}$ DTA criteria were used and suppressing dose below $10 \%$, the average and standard deviation for local dose of $90.91 \% \pm 4.41$ and for maximum dose of gamma index are $92.48 \pm$ 3.20 .

The same gamma criteria used in 2D-array measurements are applied using Gafchromic EBT3 films. The agreements for local doses are $80.19 \pm 4.73$, for maximum dose $88.45 \pm 4.04$. The data indicate also a better performance for $2 \mathrm{D}$-array compared to films as our results.

Sancar et al. [8] prepared quality control plans for 15 patients to test clinical usability of Gafchromic EBT2 film. Gamma analysis values were found between $68.11 \%$ and $99.89 \%$ for $3 \%$ DD and $3 \mathrm{~mm}$ DTA criteria, and they had quite variable values. They stated that best results they obtained were at small fields.

Spezi et al. [19] showed that repeatability of PTW 2D-ARRAY seven 29 was very successful even in small regions for short, medium and large term by measuring at open spaces with size from $2 \times 2 \mathrm{~cm}^{2}$ to $27 \times 27 \mathrm{~cm}^{2}$. It was indicated that array is independent from energy and it can be used for IMRT plans involving small areas.
In comparison between dose distributions obtained from TPS, and results of PTW 2D-Array seven29 and Gafchromic EBT3 film dosimetry for $3 \%$ DD and $3 \mathrm{~mm}$ DTA criteria, which was accepted as optimum criteria, it showed that while the suitability of both methods more than $90 \%$, doses measured with PTW 2D-Array seven 29 were closer than film measurements to TPS doses and this difference was statistically significant. If the range of criteria is increased, the consistency reaches to $100 \%$. Study results showed similarity by the literatures. Wide accordance (68\%-99\%) to the literature was notified particularly for film dosimetry.

\section{Conclusions}

It was observed that there is no statistically significant difference between analyzing by measuring all fields composing IMRT at the same time and analyzing by measuring each field separately. Simultaneous irradiation of the fields composing the plan will be time saving for busy clinics.

In conclusion, PTW 2D-Array seven 29 two dimensional ionization chamber systems give the result values closer to the calculated values and it does not require additional cost. Besides, 2D-Array two dimensional ionization chamber systems can be preferred due to the reasons that film dosimetry has calibration problems for scanning and uncertainties' in itself, it takes time to use and it has large cost. The gamma criterion of $3 \% / 3 \mathrm{~mm}$ was selected as the most suitable criteria for routine prostate IMRT quality assurance in our clinic.

\section{References}

1. Alber M, Broggi S, De Wagter C, Eichwurzel I, Engström P, et al. (2008) Guidelines for the verification of IMRT. ESTRO. Brussels, Belgium.

2. Ezzell GA, Galvin JM, Low D, Palta JR, Rosen I, et al. (2003) Guidance document on delivery, treatment planning, and clinical implementation of IMRT: report of the IMRT Subcommittee of the AAPM Radiation Therapy Committee. Med Phys 30: 2089-2115.

3. Saminathan S, Manickam R, Chandraraj V, Supe SS (2010) Dosimetric study of $2 \mathrm{D}$ ion chamber array matrix for the modern radiotherapy treatment verification. J Appl Clin Med Phys 11: 3076.

4. Li JG, Yan G, Liu C (2009) Comparison of two commercial detector arrays for IMRT quality assurance. J Appl Clin Med Phys 10: 2942.

5. Poppe B, Blechschmidt A, Djouguela A, Kollhoff R, Rubach A, et al. (2006) Two-dimensional ionization chamber arrays for IMRT plan verification. Med Phys 33: 1005-1015.

6. Palmer AL, Nisbet A, Bradley D (2013) Verification of high dose rate brachytherapy dose distributions with EBT3 Gafchromic film quality control techniques. Phys Med Biol 58: 497-511.

7. McDermott LN, Wendling M, van Asselen B, Stroom J, Sonke JJ, et al. (2006) Clinical experience with EPID dosimetry for prostate IMRT pretreatment dose verification. Med Phys 33: 3921-3930.

8. Sancar A, Ayyangar KM, Nehru RM, Kurup PG, Murali V, et al. (2006) Comparison of Kodak EDR2 and Gafchromic EBT film for intensitymodulated radiation therapy dose distribution verification. Med Dosim. 31: 273-82.

9. Guerda Massillon-JL, Sou-Tung Chiu-Tsao, Ivan Domingo-Munoz, Maria F. Chan (2012) Energy Dependence of the New Gafchromic EBT3 Film: Dose Response Curves for $50 \mathrm{KV}, 6$ and 15 MV X-Ray Beams. International Journal of Medical Physics, Clinical Engineering and Radiation Oncology 1: 60-65.

10. Llinares AU, Fernandez AS, Pain AE, Cabalerro LJR, Oquendo MI (2012) Ep-1319 octavius phantom and Seven29 2D array in IMRT patient verification. Technical and clinical organization aspects. Radiotherapy and Oncology 103: 500-501. 
Citation: Nalbant N, Kesen ND, Hatice B (2014) Pre-Treatment Dose Verification of Imrt Using Gafchromic Ebt3 Film and 2d-Array. J Nucl Med Radiat Ther 5: 182. doi:10.4172/2155-9619.1000182

Page 6 of 6

11. Herzen J, Todorovic M, Cremers F, Platz V, Albers D, et al. (2007) Dosimetric evaluation of a 2D pixel ionization chamber for implementation in clinical routine. Phys Med Biol 52: 1197-1208.

12. Low DA, Dempsey JF (2003) Evaluation of the gamma dose distribution comparison method. Med Phys 30: 2455-2464.

13. Low DA, Harms WB, Mutic S, Purdy JA (1998) A technique for the quantitative evaluation of dose distributions. Med Phys 25: 656-661.

14. Ezzell GA, Burmeister JW, Dogan N, LoSasso TJ, Mechalakos JG, et al. (2009) IMRT commissioning: multiple institution planning and dosimetry comparisons, a report from AAPM Task Group 119. Med Phys 36: 5359-5373.

15. Bohsung J (2004) The 2D-Array Seven29 A new way of dosimetric verification of IMRT beams, Application Note. PTW Frieburg, Berlin.
16. Saminathan S, Manickam R, Chandraraj V (2011) Plan evaluation and dosimetric comparison of IMRT using AAPM TG119 test suites and recommendations. Australas Phys Eng Sci Med 34: 55-61.

17. Gelosa S, Monti AF, Berlusconi C, Cappelletti P, Frigerio M (2009) Is gamma index evaluation for IMRT pre-treatment QA affected by gantry angles? Radiotherapy and Oncology 92: 214.

18. Elawady RA, Attalla EM, Elshemey WM, Shouman T, Alsayed AA (2014) Dose verification of intensity modulated radiotherapy in head and neck tumors. Int J Cancer Ther Oncol 2: 02037.

19. Spezi E, Angelini AL, Romani F, Ferri A (2005) Characterization of a 2D ion chamber array for the verification of radiotherapy treatments. Phys Med Biol 50: 3361-3373. 